\title{
Calidad de vida en pacientes con rinitis alérgica: estudio comparativo con la hipertensión arterial en el ámbito de atención primaria
}

\section{Quality of life in patients with allergic rhinitis: comparative study with hypertension in primary care}

\author{
B. de la Hoz ${ }^{1}$, C. Colás ${ }^{2}$, M. Rodríguez Rodríguez ${ }^{3}$, Grupo Freedom*
}

\section{RESUMEN}

Objetivo. Estudiar la calidad de vida de los pacientes con rinitis alérgica en el ámbito de la atención primaria.

Material y métodos. Estudio observacional, transversal, multicéntrico a nivel estatal. Se estudió una muestra de pacientes con rinitis alérgica (RA) y como grupo control, pacientes con hipertensión arterial. Ambos grupos respondieron a un cuestionario general de las características demográficas y actividades habituales, el cuestionario genérico de calidad de vida SF-12 y el cuestionario específico de calidad de vida mini-RQLQ.

Resultados. Participaron 133 investigadores que incluyeron en el estudio 1.309 pacientes; de ellos $1.093(83,56 \%)$ presentaban RA y $215(16,44 \%)$ HTA. La RA da lugar a una afectación significativamente mayor sobre la actividad física, el trabajo, la conducción y el ocio que la HTA. Los dos grupos de pacientes mostraron un deterioro en la calidad de vida relacionada con la salud (CVRS), medido por el SF12, sin objetivarse diferencias estadísticas significativas entre ambos grupos. Las medias ajustadas por edad y sexo del Componente Sumario Físico (CSF-12) fueron $54,2 \pm 19,30$ para la RA y $57,6 \pm 22,01$ para el grupo con HTA $(p=0,075)$. Las medias ajustadas por edad y sexo para el Componente de Sumario Mental (CSM) fueron de $56,9 \pm 19,70$ para RA y $58,8 \pm 19,82$ para la HTA $(p=0,33)$. En los pacientes con RA la calidad de vida está afectada en todos los dominios del cuestionario específico mini-RQLQ, sobre todo en el correspondiente a los síntomas nasales. La relación entre los resultados del mini-RQLQ y la repercusión de la calidad de vida en cuanto a las actividades cotidianas fue buena. El porcentaje de pacientes con RA que presentaba una enfermedad concomitante fue del 76,7\%, La patología asociada con mayor frecuencia fue el asma. Conclusiones. Los pacientes con RA atendidos en atención primaria presentan un impacto moderado sobre la CVRS medido mediante un cuestionario genérico. La afectación de CVRS es similar en pacientes con RA e HTA, a pesar de que los pacientes con RA muestran, significativamente, una mayor afectación de las actividades cotidianas. La frecuente comorbilidad de los pacientes con RA obliga a evaluar la presencia de otras patologías alérgicas especialmente el asma.

Palabras clave. Calidad de vida relacionada con la salud. Cuestionario SF-12. Cuestionario RQLQ. Rinitis.

An. Sist. Sanit. Navar. 2009; 32 (2): 169-181

1. Servicio de Alergología. Hospital Universitario Ramón y Cajal. Madrid.

2. Servicio de Alergología. Hospital Universitario Clínico. Zaragoza.

3. Servicio de Alergología. Hospital Universitario Príncipe de Asturias. Alcalá de Henares. Madrid.

* Colaboradores del estudio. Grupo Freedom: Ignacio Antepara Ercoreca, Viki Cardona, Javier Contreras, Fernando Florido, Ramón Lleonart Bellfill, Ana Navarro Pulido, Angel Navarro, Antonio Parra, Joaquín Quiralte, Carmen Pérez Francés.

\begin{abstract}
Background. This study was conducted to determine the impact of allergic rhinitis (AR) on health-related quality of life in patients in primary care.

Methods. Observational, cross-sectional, multicentre study that included a national sample of patients consulting general practitioners for AR. Patients consulting for Hypertension were studied as a control sample. A general activities questionnaire, the mini Rhino conjunctivitis Quality of Life Questionnaire (mini-RQLQ) and the Medical Outcome Short-Form (SF-12) were employed.

Results. A total of 133 general practitioners and 1,309 patients were included, 1,093 (83.56\%) patients were diagnosed as AR and 215 (16.44) as Hypertensive. Adjustments were made for age and gender. The effect of AR on daily activities was noteworthy; the impairment in activities in relationship with sport, work, driving cars and entertainment was higher in AR than in patients with hypertension, with statistical differences. Both diseases, AR and hypertension, had a moderate impact on SF-36 physical and mental summary scores, without statistical significance. Adjusted sex and age were PCS12: (AR: $54.2 \pm 19.30$; hypertension 57.6 $\pm 22.01, \mathrm{p}=0.075)$ and MCS12:(AR: $56.9 \pm 19.70$; hypertension 58.8 $\pm 19.82, \mathrm{p}=0.330$ ), without statistical significance. Patients with AR showed an impact in all domains of mini-RQLQ and the severity of disease according mini-RQLQ score had a good correlation with the impairment of daily activities. $76.7 \%$ of patients with $\mathrm{AR}$ had a coexistent atopic disease (asthma, conjunctivitis, atopic dermatitis) as well as a disease in relationship with rhinitis (sinusitis, upper and lower airway infections...). Asthma has been the disease more frequently associated to AR.

Conclusion. AR and hypertension had a moderate impact on health related quality of life. It was similar in both diseases. An epidemiological association between rhinitis and asthma has been established in patients consulting primary care doctors.
\end{abstract}

Key words. Quality of life. SF-12. RQLQ. Rhinitis. Primary care.

\section{Correspondencia:}

María Belén de la Hoz Caballer

Servicio de Alergología

Hospital Ramón y Cajal

Carretera Colmenar Km. 9,100

28034 Madrid

Tfno. 913368000-Ext. 7895

Fax. 913368693

E-mail: bhoz.hrc@salud.madrid.org

Recepción: 2 de diciembre de 2008

Aceptación provisional: 30 de enero de 2009

Aceptación definitiva: 6 de mayo de 2009 


\section{INTRODUCCIÓN}

La rinitis alérgica (RA) se define clínicamente como un trastorno sintomático de la nariz, inducido por la exposición a alergenos, que ocasionan una inflamación de la mucosa nasal mediada por IgE; es un problema global de salud, que afecta entre el 5-50\% de la población y cuya prevalencia va en aumento ${ }^{1}$.

La comorbilidad de la RA con asma, conjuntivitis, dermatitis atópica e incluso alergia a los alimentos está bien documentada ${ }^{1-4}$. Hasta el $20 \%$ de los pacientes que consultan por rinitis padecen simultáneamente asma bronquial y casi la mitad conjuntivitis ${ }^{1}$. La rinitis alérgica constituye un factor de riesgo para la incidencia del asma ${ }^{2,3}$. El paralelismo epidemiológico, etiológico y funcional constatado entre las enfermedades alérgicas, en particular entre rinitis y asma ${ }^{5}$, hace prioritario el abordaje integral del paciente, siendo por ello importante efectuar un diagnóstico precoz, preciso y completo que abarque criterios clínicos, inmunológicos y evaluación funcional completa que incluya patologías concomitantes y repercusiones en la calidad de vida ${ }^{6}$.

La rinitis, aunque no es una patología de riesgo vital, supone un notable impacto en la calidad de vida, al alterar la vida social y afectar el rendimiento escolar y laboral $^{7-9}$, sirviendo este deterioro en la calidad de vida como parámetro para definir la intensidad de la enfermedad ${ }^{8}$. Además, al ser una patología muy prevalente en la población joven, la disminución de la calidad de vida conlleva no sólo alteración de la vida social y de la autoestima, sino alteración de la vida laboral y escolar con absentismo, pérdida de la productividad y concentración e incluso menor capacidad de aprendizaje $\mathrm{e}^{7-10}$. Aunque esta pérdida en la calidad de vida se supone aumentada en los pacientes que junto con rinitis asocian asma, no ha sido adecuadamente verificado.

La mayor parte de los trabajos que se han llevado a cabo para evaluar la calidad de vida en la rinitis alérgica, se han reali- zado en población atópica procedente de las consultas especializadas, de hecho la calidad de vida se ha utilizado como una útil variable de eficacia en varios ensayos clínicos con fármacos ${ }^{9,11}$. Se ha publicado un estudio con carácter general realizado en población infantil ambulatoria ${ }^{12}$ y sólo se ha comparado con otra enfermedad el asma en términos de impacto de la calidad de vida ${ }^{13}$, de manera, que en la rinitis alérgica grave el impacto sobre la calidad de vida es incluso mayor que el ocasionado por el asma ${ }^{13}$. La carencia de estudios epidemiológicos sobre la repercusión en la calidad de vida en pacientes con RA en atención primaria de nuestro país, ha motivado el planteamiento del presente estudio. Se ha utilizado como control la hipertensión arterial, por las características similares de esta entidad con la RA en cuanto a su alta prevalencia y morbilidad, pero con la diferencia de que la HTA conlleva una alta mortalidad asociada y no la RA. Por otra parte se conoce que en estudios poblacionales el impacto de la HTA sobre la calidad de vida es entre leve a moderado ${ }^{14}$. Los síntomas en la HTA tienen mayor impacto en la calidad de vida relacionada con la salud (CVRS) medida por el test SF36 que las características de los pacientes ${ }^{15}$.

El objetivo principal de este estudio ha sido evaluar la afectación de la calidad de vida de los pacientes sintomáticos con rinitis alérgica, tratados o no tratados, comparados con un grupo de pacientes hipertensos, utilizando como instrumentos cuestionarios genéricos y específicos. La hipótesis de trabajo es que la RA tiene un impacto al menos igual si no mayor que la HTA sobre la calidad de vida.

Como objetivo secundario se ha investigado la comorbilidad de la rinitis alérgica en los pacientes atendidos en el ámbito de atención primaria.

\section{MATERIAL Y MÉTODOS}

Se seleccionaron pacientes consecutivos que acudieron a su centro de salud para consultar por rinitis RA o por HTA, 
constituyendo estos últimos el grupo de comparación.

Se incluyeron los pacientes de edad igual o superior a 18 años, que acudían a consulta (primera consulta o sucesivas), de atención primaria por rinitis alérgica sintomática con independencia de que estuvieran o no tratados, o con hipertensión arterial mal controlada y que otorgaban consentimiento informado. Fueron excluidos aquellos pacientes que padecían poliposis nasal o que presentaban asociación de RA e HTA.

Se definió caso de RA aquella rinitis clínica sintomática con pruebas cutáneas positivas a aeroalergenos comunes en nuestro medio (pólenes, ácaros, hongos, etc.) con o sin tratamiento. No se consideraron imprescindibles las pruebas cutáneas si la sintomatología nasal se había presentado con las mismas características, en el mismo periodo anual, al menos 2 años consecutivos. A los pacientes que tuvieran una puntuación $\geq 4$ para síntomas nasales u oculares en el cuestionario miniRQLQ, se consideró que tenían una RA grave y se analizó como subgrupo.

Se definió caso de HTA a aquel paciente que en el momento de la consulta presentaba una tensión arterial igual o superior a 140 sistólica $/ 90 \mathrm{~mm} \mathrm{Hg}$ diastólica.

La selección de pacientes se realizó en las condiciones de actuación médica habituales y de acuerdo a la práctica clínica. El periodo de inclusión se estableció del 1 de abril al 30 de junio de 2004.

Se diseñó un estudio observacional, transversal y multicéntrico, en el que los pacientes incluidos contestaron a los siguientes cuestionarios:

- Cuestionario SF-12 ${ }^{16,17}$, cuestionario genérico para medir CVRS en pacientes con RA y HTA. El cuestionario SF-12 es la versión reducida del SF-36; está formado por un subconjunto de 12 ítems del SF-36, seleccionados mediante regresión lineal múltiple (se seleccionaron uno o dos ítems de cada una de las dimensiones del SF-36) a partir de los cuales se construyen los componentes sumarios físico (CSF-12) y mental (CSM-12) del SF-12 como únicas puntuaciones.

- Cuestionario mini-RQLQ (Rhinoconjunctivitis Quality of Life Questionnaire $)^{18}$, cuestionario específico para medir CVRS en pacientes con RA, validado y utilizado en ensayos clínicos de RA como variable de eficacia.

- Cuestionario diseñado para este estudio en el que se realizaban por el médico al paciente las siguientes preguntas cerradas con el siguiente esquema:

1. Variables demográficas (edad, sexo), hábitat (urbano/rural), nivel de estudios (básico/medio/ diplomado/universitario).

2. Actividades cotidianas. Actividad física o deporte (sí/no), grado de actividad física o deporte (bajo/ medio/alto), tipo de trabajo (físico/intelectual/intermedio), valoración del estrés (presencia/ausencia), actividades específicas (conducción: sí/no).

3. Valoración de repercusión subjetiva de la enfermedad RA o HTA sobre: la actividad física, el trabajo, el ocio, la conducción y el estrés. Con las alternativas sí o no.

4. El médico recogía la enfermedades concomitantes alérgicas o relacionadas: sinusitis, otitis, infecciones respiratorias de vía aérea superior e inferior (IVRS, IVRI), asma, conjuntivitis, eczema, urticaria, dermatitis, alergia a alimentos, alergia a fármacos. Con las alternativas: sí, no, no sabe, no contesta.

\section{Análisis estadístico}

Las características de los pacientes se describieron utilizando la media, mediana y desviación estándar en el caso de variables continuas (Ej. puntuación mini- 
RQLQ) y las variables categóricas se describieron mediante frecuencias y porcentajes (Ej. sexo).

Las comparaciones de las variables categóricas entre los distintos grupos de pacientes se realizaron mediante el test Chi-Cuadrado. Para la comparación de variables continuas en las que fueron necesarias pruebas no paramétricas se utilizó el test de Mann-Whitney.

Para valorar la calidad de vida de los pacientes se utilizó la Escala de Calidad de Vida SF-12. Se realizó un análisis ajustado por edad y sexo de las puntuaciones medias en las medidas sumario físico y mental, comparándose las puntuaciones de los pacientes con rinitis alérgica e hipertensión mediante el análisis de la covarianza (ANCOVA).

Para evaluar la relación existente entre las puntuaciones del cuestionario miniRQLQ y las obtenidas en el CSF y CSM en pacientes con RA se utilizó la correlación de Pearson.

Las pruebas estadísticas fueron bilaterales y se consideraron significativas cuando los p-valores fueron inferiores a 0,05 . Los datos fueron analizados con el programa informático SAS, versión 8,2.

Para la estimación del tamaño de muestra para ambos grupos, a través de las puntuaciones del SF-12, se asumieron una desviación típica igual para ambos grupos de $\sigma=20$, y se consideró una diferencia significativa entre los dos grupos un $5 \%$. Se consideró un nivel de confianza (1- $\alpha$ ) en un $95 \%, \alpha=0,5$ y potencia de 0,8 (1$\beta), \beta=0,2$. El tamaño estimado fue de 1.776 pacientes, repartidos un 75\% en RA: 1.332 pacientes y un 25\% en HTA: 444 pacientes.

\section{RESULTADOS}

En el estudio participaron 133 investigadores de atención primaria que reclutaron 1.493 pacientes de los cuales 1.308 un $87,61 \%$ fueron aptos para el estudio tras aplicar los criterios de inclusión y exclusión. De estos pacientes $1.093(83,56 \%)$ presentaban RA y 215 (16,44\%) HTA. En la distribución geográfica de la procedencia de los pacientes no se encontraron diferencias significativas respecto a la distribución geográfica de la población española (censo de población del instituto de estadística. julio 2002).

\section{Comparación de variables demográficas y actividades cotidianas entre los pacientes con RA E HTA}

En el análisis de homogeneidad de los dos grupos de estudio se encontraron diferencias significativas entre los dos grupos de estudio RA y HTA para la edad y para el sexo: eran más jóvenes los pacientes con RA (Media = 38 Desviación estándar $=14,45)$ que los pacientes con HTA (Media=58, Desviación estándar=13,23) y existía mayor porcentaje de mujeres en los pacientes con RA (50\% de mujeres) que en HTA ( $41 \%$ de mujeres). Por ello todo el análisis estadístico se ajustó en función de estas dos variables. No se encontraron diferencias significativas en función del tipo de enfermedad RA e HTA para las variables estudiadas hábitat, nivel de estudios, hábitos tóxicos, actividad física y deporte, tipo de trabajo, estrés y conducción (Tabla 1). 
Tabla 1. Características de la muestra según tipo de enfermedad y variables.

\begin{tabular}{|c|c|c|c|}
\hline Variables & $\mathbf{R A}$ & HTA & Comparación § \\
\hline Edad (media, desviación estándar) & $(38 / 14,45)$ & $58 / 13,23$ & $\mathrm{P}<0,001$ \\
\hline Sexo (\% mujeres) & 50 & 51 & $\mathrm{P}=0,04$ \\
\hline Habitat (\% pacientes rural) & 19 & 21 & ns \\
\hline $\begin{array}{l}\text { Tipo de trabajo (\% pacientes) } \\
\text { Físico } \\
\text { Intermedio } \\
\text { Intelectual }\end{array}$ & $\begin{array}{l}27,32 \% \\
35,06 \% \\
37,57 \% \\
\end{array}$ & $\begin{array}{l}45,06 \% \\
32,10 \% \\
22,84 \% \\
\end{array}$ & $\mathrm{~ns}^{*}$ \\
\hline $\begin{array}{l}\text { Nivel de estudios* (\% pacientes) } \\
\text { Básico } \\
\text { Medio } \\
\text { Diploma } \\
\text { Universitario }\end{array}$ & $\begin{array}{l}26,32 \% \\
37,95 \% \\
15,65 \% \\
20,00 \%\end{array}$ & $\begin{array}{c}48,52 \% \\
26,04 \% \\
8,88 \% \\
16,50 \%\end{array}$ & $\mathrm{~ns}^{* *}$ \\
\hline Actividad física o deporte** (\% pacientes sí) & 45 & 30 & $\mathrm{~ns}^{*}$ \\
\hline $\begin{array}{l}\text { Grado actividad física/ deporte (\% pacientes) } \\
\text { Bajo } \\
\text { Medio } \\
\text { Alto }\end{array}$ & $\begin{array}{l}24,36 \% \\
65,38 \% \\
10,26 \%\end{array}$ & $\begin{array}{c}38,00 \% \\
56,00 \% \\
6,00 \%\end{array}$ & ns \\
\hline Conducción (\% pacientes que sí conducen) & 62,44 & 56,86 & ns \\
\hline $\begin{array}{l}\text { Estrés (\% pacientes) } \\
\text { No } \\
\text { Algo } \\
\text { Sí }\end{array}$ & $\begin{array}{l}30,22 \% \\
52,00 \% \\
17,69 \%\end{array}$ & $\begin{array}{l}30,49 \% \\
46,34 \% \\
23,78 \%\end{array}$ & ns \\
\hline Manejo de maquinaria (\% pacientes que sí manejan) & 10,59 & 18,92 & $\mathrm{p}=0,04$ \\
\hline
\end{tabular}

$\S$ prueba chi-cuadrado

t- Student

*ns ajustado por edad ** ns ajustado por edad y hábitat

Comparación de la calidad de vida en los pacientes con rinitis alérgica y HTA

Del total de 1.308 pacientes, 903 (69\%) tenían información recogida para el cuestionario SF-12. De estos pacientes 732 (81\%) correspondían a RA y 170 (19\%) a HTA. El cuestionario SF-12 fue evaluado para los dos grupos de pacientes y comparados los resultados obtenidos en cada uno de los dominios del cuestionario, no encontrándose diferencias significativas para ninguno de los dominios del SF-12 entre las dos enfermedades (Tabla 2). 
Tabla 2. Resultados de la comparación de la calidad de vida relacionada con la salud en pacientes con RA y HTA. Cuestionario SF-12.

\begin{tabular}{|c|c|c|c|c|c|c|c|}
\hline CSF-12 & Media & DE & ES & 95\% IC superior & 95\% IC inferior & Mediana & Comparación* \\
\cline { 1 - 6 } RA (n=732) & 54,02 & 19,3 & 0,7 & 53,66 & 56,47 & 52,5 & \multirow{2}{*}{ P $=0,075$} \\
\hline HTA (n=170) & 57,60 & 22,0 & 1,7 & 49,18 & 55,85 & 52,5 & \\
\hline CSM-12 & Media & DE & ES & 95\% IC superior & 95\% IC inferior & Mediana & Comparación* \\
\hline RA (n=732) & 56,9 & 19,70 & 0,8 & 55,78 & 58,64 & 55,16 & \multirow{2}{*}{ P $=0,33$} \\
\hline
\end{tabular}

*Puntuaciones ajustadas por edad y sexo. Análisis de la covarianza (ANCOVA)

DE: Desviación estándar

ES: Error estándar

IC: Intervalo de confianza

CSF-12: Componente Sumario Físico. SF-12

CSM-12: Componente Sumario Mental. SF-12

Del total de 1.093 pacientes con RA, 742 $(67,89 \%)$ pacientes tenían recogida la información del cuestionario mini-RQLQ. La media obtenida para todos los dominios de evaluación de la CVRS estaba situada entre "algo afectado" y "moderadamente afectado" (Tabla 3).

Tabla 3. Resultados de cuestionario calidad en rinitis mini -RQLQ de 742 pacientes.

\begin{tabular}{lcccc}
\hline \multicolumn{1}{c}{ Variables } & Media & DE & $\mathbf{9 5 \%}$ IC & Mediana \\
\hline Actividades & 2,51 & 1,26 & $2,42-2,60$ & 2,67 \\
Problemas locales & 3,17 & 1,23 & $3,09-3,26$ & 3,00 \\
Síntomas nasales & 3,62 & 1,26 & $3,52-3,71$ & 3,67 \\
Síntomas oculares & 2,36 & 1,27 & $2,27-2,45$ & 2,33 \\
Otros síntomas & 2,03 & 1,34 & $1,94-2,13$ & 2,00 \\
Global Mini-RQLQ & 2,71 & 1,03 & $2,63-2,78$ & 2,71 \\
\hline
\end{tabular}

DE: Desviación estándar IC: Intervalo de confianza

Los pacientes con RA e HTA en las actividades cotidianas no presentan diferencias significativas en cuanto al porcentaje de pacientes que las realiza, si bien se encontraron diferencias al analizar la repercusión subjetiva sobre las actividades cotidianas, que refieren los pacientes en relación con el tipo de enfermedad. Los pacientes afectos de RA atribuyen a esta patología una limitación significativamente mayor sobre: su nivel de actividad física en general y deporte, actividad laboral, actividades de ocio y conducción de vehículos, que la que los pacientes con HTA atribuyen a la propia enfermedad, de forma independiente de la edad y sexo. Únicamente se establece una valoración similar para ambas patologías en la capacidad de generar estrés (Tabla 4). 
Tabla 4. Afectación sobre actividades cotidianas comparación entre rinitis alérgica (RA) e hipertensión arterial (HTA).

\begin{tabular}{lcc}
\hline \multicolumn{1}{c}{ Variable } & \% Pacientes Sí afectación & Significación $(\gamma 2)$ \\
\hline Afectación Actividad física y deportes & $\mathrm{RA}=63 \%$ & $\mathrm{P}=0,001^{*}$ \\
Afectación Trabajo & $\mathrm{HTA}=41 \%$ & $\mathrm{P}=0,001^{*}$ \\
& $\mathrm{RA}=62 \%$ & $\mathrm{P}=0,0001^{*}$ * \\
Afectación Ocio & $\mathrm{HTA}=35 \%$ & $\mathrm{P}=0,001^{* *}$ * \\
& $\mathrm{RA}=56 \%$ & \\
Afectación Conducción & $\mathrm{HTA}=26 \%$ & $\mathrm{NS}$ \\
\end{tabular}

Prueba de $\gamma 2 .{ }^{*}$ Ajustado por edad y sexo. ${ }^{* *}$ Ajustada por edad. ${ }^{* * *}$ Ajustada por sexo

Se objetiva una correlación inversa (por el sistema de puntuación) débil entre las puntuaciones globales de los compo- nentes sumarios físico (CSF-12) y mental (CSM-12) y la puntuación global del miniRQLQ (Tabla 5).

Tabla 5. Correlación entre las puntuaciones del SF-12 y el Mini-RQLQ pacientes con rinitis alérgica.

\begin{tabular}{|c|c|c|c|c|c|c|}
\hline \multirow{2}{*}{$\begin{array}{l}\text { Gravedad de RA } \\
\text { Cuestionario }\end{array}$} & \multicolumn{2}{|c|}{$\mathbf{R A}$} & \multicolumn{2}{|c|}{ RA-Grave } & \multicolumn{2}{|c|}{ RA-No Grave } \\
\hline & SF-12 Físico & SF-12 Mental & SF-12 Físico & SF-12 Mental & SF12 Físico & SF12 Mental \\
\hline MINI-RQLQ & $-0,40$ & $-0,27$ & $-0,25$ & $-0,17$ & $-0,40$ & $-0,29$ \\
\hline Significación & $\mathrm{P}<0,001$ & $P=0,001$ & $\mathrm{P}<0,001$ & $P=0,001$ & $\mathrm{P}<0,001$ & $\mathrm{P}<0,001$ \\
\hline
\end{tabular}

Correlación de Pearson (N=731)

\section{Análisis de un subgrupo de pacientes con rinitis alérgica grave}

De los 742 pacientes con RA un subgrupo de $374(50,40 \%)$ presentó una RA grave (puntuación de síntomas nasales u oculares en Mini-RQLQ mayor de 4) el resto se consideró $\mathrm{RA}$ no grave. $\mathrm{Al}$ comparar este grupo de pacientes con RA grave con los pacientes HTA mediante el cuestionario SF-12, no se encontraron diferencias significativas en ninguno de los dominios de SF-12. Se encuentran diferencias estadísticamente significativas en las puntuaciones del SF12 entre los pacientes con RA grave y no grave. La puntuación del CSF-12 y CSM-12 son menores en los pacientes con RA grave que en el resto de los pacientes de la muestra de RA (Tabla 6). 
Tabla 6. Resultados de la comparación de la calidad de vida relacionada con la salud en pacientes con RA No grave y RA grave. Cuestionario SF-12.

\begin{tabular}{|c|c|c|c|c|c|c|}
\hline CSF-12 & Media & DE & $95 \%$ IC inferior & $95 \%$ IC superior & Mediana & Comparación* \\
\hline RA No grave $(n=364)$ & 56,03 & 19,83 & 53,01 & 58,40 & 52,5 & \multirow{2}{*}{$\mathrm{P}<0,001$} \\
\hline RA grave $(\mathrm{n}=368)$ & 50,45 & 18,74 & 48,53 & 52,36 & 52,5 & \\
\hline CSM-12 & Media & DE & $95 \%$ IC inferior & 95\% IC superior & Mediana & Comparación* \\
\hline RA No grave (n=364) & 58,81 & 19,70 & 56,29 & 59,91 & 55,5 & \multirow{2}{*}{$\mathrm{P}<0,01$} \\
\hline RA grave $(n=368)$ & 54,16 & 18,87 & 52,23 & 56,08 & 53,5 & \\
\hline
\end{tabular}

* Mann-Whitney. DE: Desviación estándar. IC: Intervalo de confianza. CSF-12: Componente Sumario Físico. SF-12

CSM-12: Componente Sumario Mental. SF-12

Al comparar los dos grupos de pacientes con RA grave y RA no grave se encontró un porcentaje mayor de pacientes que referían impacto sobre las actividades cotidia- nas y el trabajo en los pacientes con rinitis alérgica grave respecto a los no graves con diferencias significativas (Tabla 7).

Tabla 7. Afectación sobre las actividades cotidianas. Comparación de la rinitis alérgica grave y no grave.

\begin{tabular}{llc}
\hline \multicolumn{1}{c}{ Variable } & \% P SI afectación & Comparación \\
\hline Afectación Actividad física & RA-Grave $=71,47$ & $\gamma 2=20,48$ \\
& RA-No Grave $=54,11$ & $\mathrm{P}=0,0001$ \\
Afectación Trabajo & RA-Grave $=69,35$ & $\gamma 2=19,2$ \\
& RA-No Grave $=55,49$ & $\mathrm{P}=0,0003$ \\
Afectación Ocio & RA-Grave $=64$ & $\gamma 2=17,68$ \\
& RA-No Grave $=47,52$ & $\mathrm{P}=0,0001$ \\
& & $\gamma 2=7,8$ \\
Afectación Conducción & RA-Grave $=37,34$ & $\mathrm{P}=0,005$ \\
& RA-No Grave $=27$ & $\gamma 2=28,61$ \\
Afectación Estrés & RA-Grave $=55,56$ & $\mathrm{P}=0,0001$ \\
& RA-No Grave $=34,38$ & \\
\end{tabular}

\%: porcentaje de pacientes RA-Grave $=\mathrm{n}^{\mathrm{o}}$ pacientes 374 ; RA-No Grave $=\mathrm{n}^{\mathrm{o}}$ pacientes 368 total con cuestionario mini-RQLQ $=742$

\section{Comorbilidad de la rinitis alérgica}

De los 1.093 pacientes con RA un total de 688 pacientes (63\%) presentaban alguna enfermedad concomitante. De los 688 un $21 \%$ presentaba 3 enfermedades concomitantes. Las más frecuentes fueron: conjuntivitis y asma (Fig. 1). 


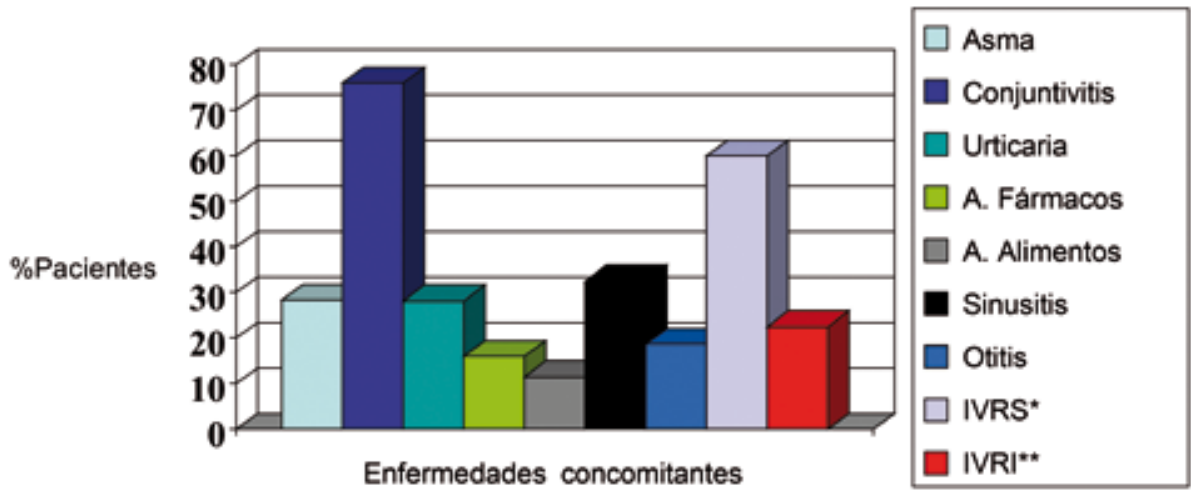

*IVS: Infección via respiratoria superior

*IVRI: Infección vía respiratoria inferior

Figura 1. Conmorbilidad Rinitis Alérgica.

\section{DISCUSIÓN}

En el ámbito médico, la rinitis alérgica ha sido infravalorada al ser considerada como una patología carente de riesgo vital. Esta apreciación ha cambiado en la última década, tras mostrar los estudios realizados sobre la CVRS, que los pacientes con rinitis tenían una afectación global e importante de la CVRS, lo que la ha hecho objeto de una consideración especial.

La repercusión en la calidad de vida de la rinitis alérgica se ha explorado mediante cuestionarios genéricos, el más utilizado el SF36 (Medical Outcomes Survey Short Form 36) y su versión breve el SF-12 $2^{11,19}$ y cuestionarios específicos entre los cuales el más conocido es el RQLQ (Rhinoconjunctivitis Quality of Life Questionnaire) que está traducido y validado en español, y sus diversas versiones, entre ellas el mini -RQLQ ${ }^{7,8,18,20,21}$. En 2007 se ha publicado un nuevo cuestionario específico para $\mathrm{RA}^{22} \mathrm{de}-$ sarrollado íntegramente en España que ha demostrado muy buenas propiedades psicometrías y es de gran utilidad para medir calidad de vida en pacientes con RA españoles, además se dispone de una versión abreviada de 15 ítems ${ }^{23}$.
El SF-36, cuestionario genérico más utilizado en la rinitis alérgica ${ }^{11,20}$, ha demostrado el deterioro de la CVRS en pacientes con rinitis alérgica respecto a pacientes sa$\operatorname{nos}^{10,20}$ y su utilidad para evaluar el cambio en la CVRS en los pacientes con rinitis alérgica tras recibir tratamiento ${ }^{11}$. En diversas patologías alérgicas como asma, dermatitis atópica y rinitis se ha utilizado un modelo abreviado el SF-1224-27, que también se ha validado en español. Este cuestionario se considera una buena alternativa al SF36 para evaluar los componentes globales físico y mental en proyectos en los que participan un número alto de sujetos ${ }^{24-27}$, como ha sido este estudio. De igual forma, el cuestionario mini-RQLQ utilizado en este estudio, posee las mismas propiedades discriminatorias que la versión larga, y aunque su sensibilidad al cambio es menor, tiene la ventaja de su utilidad en estudios con un gran número de pacientes, como las encuestas epidemiológicas y en ensayos clínicos sobre eficiencia ${ }^{18}$.

Es muy interesante conocer el impacto en la CVRS de una determinada patología en comparación con otras enfermedades, en especial aquellas que por diversos mo- 
tivos como su prevalencia, morbilidad, mortalidad, o por su asociación a otras patologías, se consideran enfermedades relevantes para la Salud Pública y el propio individuo, no habiéndose realizado hasta la fecha estudios que comparasen la repercusión sobre la CVRS en pacientes con $\mathrm{RA}$ respecto de otras enfermedades como diabetes, hipertensión arterial, depresión, etcétera, en el ámbito de atención primaria. En este estudio las puntuaciones para el componente Sumario Físico y Mental de la muestra global de los pacientes con RA e HTA se encuentran en ambas enfermedades por encima de la media publicada para la población española, que es para ambos CSF-12 y CSM-12, una media de 50 y desviación estándar de 10, con una diferencia respecto a población general entre moderada para RA y pequeña para $\mathrm{HTA}^{28}$.

La comparación de las dos patologías rinitis alérgica e hipertensión arterial ha permitido establecer en este estudio, que ambas inciden de una manera muy similar sobre la CVRS de los pacientes en el ámbito de la atención primaria, a pesar de las diferencias de pronóstico vital que ambas enfermedades presentan. Si bien, dos importantes características demográficas, la edad y el sexo, eran diferentes en las muestras de pacientes con rinitis e hipertensión, lo que supone una limitación del estudio. No se encontraron diferencias significativas en los componentes físico y mental del SF12 entre las dos enfermedades, al corregir los resultados por edad y sexo.

Se ha explorado de forma independiente y más exhaustiva la repercusión de la enfermedad sobre ciertas actividades diarias. En este sentido se recoge en este estudio, con un cuestionario sencillo, como repercute la enfermedad en sus actividades cotidianas. Se encuentra que el porcentaje de pacientes en los que la enfermedad ocasiona mayor repercusión sobre actividades diarias, como la actividad física y deporte, el trabajo, el ocio y la conducción es mayor de forma significativa entre los pacientes con RA respecto a los que presentaban HTA, en cualquier grupo de edad, y sin diferencias respecto al sexo. Estos datos co- inciden con otros estudios que analizan la repercusión de los factores sociales sobre la calidad de vida de los pacientes con RA o asma ${ }^{27,29}$.

En un estudio en el que se comparó la HTA con la dermatitis atópica, en población general, se objetivó un deterioro mayor en las puntuaciones físicas para la HTA, pero en los dominios mental y social el deterioro fue mayor para la dermatitis atópica con una diferencia que fue estadísticamente significativa ${ }^{25}$. En el estudio actual es llamativa la repercusión sobre el ocio y el deporte que refieren los pacientes con RA, situación que puede relacionarse directamente con las características de la enfermedad, y que conlleva evitación de irritantes y aeroalergenos.

Se ha demostrado que las enfermedades alérgicas tienen un impacto negativo en la calidad de vida relacionada con la salud, que habitualmente se relaciona con la gravedad de la clínica.

Hasta ahora las puntuaciones existentes correspondían a pacientes atendidos en consulta del especialista, que presuponían mayor gravedad, no disponiéndose de la valoración realizada en atención primaria. En este estudio se ha objetivado que los pacientes con RA tienen puntuaciones mayores de CSF-12 y CSM-12 que las publicadas en estudios españoles con muestras de pacientes que son reclutados en las consultas de especialidades, en general de alergología ${ }^{23,30}$. Los resultados del mini RQLQ de este estudio muestran que las puntuaciones para cada uno de los dominios del cuestionario y la puntuación global son inferiores (menor afectación) a los resultados obtenidos en pacientes españoles remitidos a las consultas especializadas, que presentan mayores puntuaciones (mayor afectación).

En cuanto al análisis de los pacientes con RA se objetiva una correlación débil entre la puntuación global del mini-RQLQ y el CSF-12, y no correlación con el CSM, similar a la correlación que se encuentra en otros estudios en población con RA de especializada y que comparan ambos cuestionarios. 
Se ha observado una mejor relación entre el mini-RQLQ y la repercusión de la RA sobre las actividades cotidianas, que entre el mini RQLQ y el SF12. Esta situación de una mejor relación entre la repercusión de la enfermedad sobre las actividades cotidianas y los cuestionarios específicos, que entre genéricos y específicos se ha obtenido también en los estudios de calidad de vida en asma ${ }^{29}$.

En este estudio se aportan los datos de una muestra de población no seleccionada mediante criterios ARIA $^{31}$, por lo que se seleccionó un grupo de pacientes con RA considerada más grave por criterios clínicos del mini-RQLQ, resultando que casi la mitad de los pacientes incluidos referían síntomas moderados o graves. Además, estos pacientes sí tienen una repercusión mayor, estadísticamente significativa, sobre la calidad de vida que los pacientes con síntomas leves, medida por el SF-12. De igual forma, es mayor la repercusión de la RA sobre las actividades cotidianas en los pacientes con más síntomas nasales. Este hallazgo clínico se puede traducir en que el paciente afecto de rinitis realiza la consulta médica cuando los síntomas afectan CVRS, produciéndole interferencia con sus actividades cotidianas, aspecto éste que debe ser tenido en cuenta a la hora de aplicar con prontitud los recursos terapéuticos adecuados.

La evaluación de las actividades no se ha hecho con un cuestionario previamente validado. No obstante, las preguntas han sido idénticas para todos los individuos y las diferencias que existen entre los dos grupos son, cuantitativamente, muy marcadas, por lo que su valor es innegable. Especial mención requiere la limitación en la actividad de la conducción, por el riesgo para la seguridad de las personas que conlleva. La presencia de salvas de estornudos repetidos o de rinorrea incoercible puede llevar a distracciones momentáneas, de consecuencias potencialmente importantes.

Por otro lado, la limitación en la actividad laboral, también tiene mucha importancia para los pacientes, especialmente por tratarse de individuos jóvenes que es- tán consolidando su puesto en el mercado laboral y el padecimiento de esta patología puede limitar sus expectativas en ese campo. Estos datos coinciden con un estudio en pacientes con rinitis alérgica en atención primaria realizado en Francia ${ }^{32}$.

Por otra parte, aunque en estudios realizados en la población atendida en consultas de alergia es conocida la morbilidad de RA asociada con otras enfermedades como el asma, la conjuntivitis o la dermatitis atópica, se desconocía si esta circunstancia se presentaba también en una muestra de población más amplia como la que se da en el ámbito de la atención primaria. Los datos del presente trabajo muestran que la comorbilidad de la RA en atención primaria es llamativa: hasta el $76 \%$ de los casos presentaban conjuntivitis, el $28 \%$ asma y en el $21 \%$ había tres enfermedades asociadas con la RA. Resultados éstos similares a los obtenidos en población con RA valorada en atención especializada en nuestro país ${ }^{1}$.

Otras limitación que hay que destacar son la ausencia de información sobre los pacientes que rehusaron participar en el estudio, o bien los que no respondieron a los cuestionarios de calidad de vida ya que el porcentaje de respuesta podría variar según la gravedad de la enfermedad y por lo tanto el grado de gravedad estar menos representado que otro. Si bien no se ha llevado a cabo una selección de pacientes por ARIA y por lo tanto no se puede establecer los criterios de gravedad de esta guía, al aplicar un punto de corte de 4 puntos, los síntomas nasales de mini RQLQ o más, prácticamente ha repartido por partes iguales la muestra, situación que apunta a una muestra balanceada. Respecto al tamaño de la muestra, el número de pacientes reclutados está por debajo del estimado ya que el tamaño final de la muestra evaluable fue de 1.308 pacientes con 1.093 pacientes (83,56\% de la muestra) de RA y 215 pacientes $(16,44 \%$ de la muestra) de HTA. La muestra de pacientes RA es mayor incluso que la estimada previamente, ya que se había previsto un $75 \%$ y sí se ha reclutado menor número de paciente de HTA que los estimados $(16,44 \%$ de la muestra frente a 
un $25 \%$ estimado). Las diferencias no han afectado al resultado del SF-12, variable de comparación entre los pacientes con RA y HTA, ya que conocidas las desviaciones estándar para el CSF-12 y CSM-12 en ambos grupos de pacientes, con el tamaño de la muestra analizable, se consiguió detectar una diferencia entre grupos igual al 4,51\% con el mismo nivel de confianza (95\%).

En conclusión, la afectación de la CVRS en los pacientes con rinitis alérgica atendidos en atención primaria se encuentra entre algo y moderadamente afectada, según cuestionarios específicos. La afectación de CVRS es similar en pacientes con RA e HTA, aunque los pacientes con RA muestran, significativamente, una mayor afectación de las actividades cotidianas. La frecuente comorbilidad de estos pacientes obliga a interrogar sistemáticamente (e investigar, en caso necesario) sobre la presencia de otras patologías alérgicas especialmente el asma.

\section{Agradecimientos}

Este estudio ha sido parcialmente financiado con una beca de los laboratorios Recordati y avalado por la SEAIC.

\section{BIBLIOGRAFÍA}

1. Alergológica. Sociedad Española de Alergología e Inmunología Clínica. Madrid 1995.

2. De Benedictis FM, Bush A. Rhinosinusitis and asthma: epiphenomenon or casual association? Chest 1999; 115: 550-556.

3. Bousquet J, Vignola AM, Demoly P. Links between rhinitis and asthma. Allergy 2003; 58: 691-706.

4. Mattila L, Kilpelainen M, Terho EO, Koskenvuo M, Helenius H, Kalimo K. Food hypersensitivity among finnish university students: association with atopic diseases. Clin Exp Allergy 2003; 33: 600-606.

5. SIMONS FE. Allergic rhinobronchitis: the asthma-allergic rhinitis link. J Allergy Clin Immunol 1999; 104: 534-540.

6. Alvárez MJ, Olaguíbel JM, lasa E, Arroabarren E, Gómez A, Gómez B. De la rinitis al asma: ¿una o dos enfermedades? An Sist Sanit Navar 2003; 24 (Suplem 2): 49-55.
7. JUNIPER EF, GUYATt GH. Development and testing of a new measure of health status for clinical trials in rhinoconjunctivitis. Clin Exp Allergy 1991; 21: 77-83.

8. Juniper EF, Thompson AK, FERRIE PJ, RoBerts JN. Validation of the standardized version of the rhinoconjunctivitis Quality of Life Questionnaire. J Allergy Clin Immunol 1999; 104 (2 Pt 1): $364-369$.

9. BLAISS P. Important aspects in management of allergic rhinitis: compliance, cost and quality of life. Allergy Asthma Proc 2003; 24: 231-238.

10. Meltzer EO. The prevalence and medical and economic impact of allergic rhinitis in the United States. J Allergy Clin Immunol 1997; 99(6 Pt 2): S805-28.

11. Bousquet J, Duchateau J, Pignat JC, Fayol C, MarQUIS P, MARIZ S et al. Improvement of quality of life by treatment with cetirizine in patients with perennial allergic rhinitis as determined by a French version of the SF-36 questionnaire. J Allergy Clin Immunol 1996; 98: 309-316.

12. Pardos Martínez C, López Cortes V, González Pérez-Yarza E, Isanta Pomar C, Fuertes FernánDEZ-Espinar J, MERIZ Rubio J. Risk factors for asthma, allergy and bronchial hyperresponsiveness in children aged 6-8 years old. An Esp Pediatr 2001; 55: 205-212.

13. Leynaert B, Neukirch C, Liard R, Bousquet J, NeuКІRсн F. Quality of life in allergic rhinitis and asthma. A population-based study of young adults. Am J Respir Crit Care Med 2000; 162: 1391-1396.

14. Erickson SR, Williams BC, Gruppen LD. Perceived symptoms and health-related quality of life reported by uncomplicated hypertensive patients compared to normal controls. J Hum Hypertens 2001; 15: 539-548.

15. ERICKSon SR, Williams BC, Gruppen LD. Relationship between symptoms and health-related quality of life in patients treated for hypertension. Pharmacotherapy 2004; 24: 344-350.

16. Gandek B, Ware JE, Aaronson NK, Apolone G, BJORNER JB, BRAZIER JE et al. Cross-validation of item selection and scoring for the SF-12 Health Survey in nine countries: results from the IQOLA Project. International Quality of Life Assessment. J Clin Epidemiol 1998; 51: 1171-1178.

17. Vilagut G, Ferrer M, Rajmil L, Rebollo P, PerMANYER-Miralda G, Quintana JM et al. El cuestionario de salud SF-36 español: una década de experiencia y nuevos desarrollos. Gac Sanit 2005; 19: 135-150. 
18. Juniper EF, Thompson AK, Ferrie PJ, Roberts JN. Development and validation of the mini Rhinoconjunctivitis Quality of Life Questionnaire. Clin Exp Allergy 2000; 30: 132-140.

19. Ware JE JR, Sherbourne CD. The MOS 36-item short-form health survey (SF-36). I. Conceptual framework and item selection. Med Care 1992; 30: 473-483.

20. Bousquet J, Bullinger M, Fayol C, Marquis P, VAlentin B, Burtin B. Assessment of quality of life in patients with perennial allergic rhinitis with the French version of the SF-36 Health Status Questionnaire. J Allergy Clin Immunol 1994; 94: 182-188.

21. Soler R, de la Hoz B, Badia X, Mercadal J, Lozano R, Benavides A et al. Grupo Carino. Validación de la versión española del cuestionario de calidad de vida para pacientes con rinoconjuntivitis. Rev Clin Esp 2004; 204: 131-138.

22. ESPRINT Study Group and Investigators, VAlero A, Alonso J, Antepara I, Baró E, Colas C, Del Cuvillo A et al. Development and validation of a new Spanish instrument to measure health-related quality of life in patients with allergic rhinitis: the ESPRINT questionnaire. Value Health 2007; 10: 466-477.

23. Valero A, Alonso J, Antépara I, Baró E, Colás C, DEL Cuvillo A et al. Health-related quality of life in allergic rhinitis: comparing the short form ESPRINT-15 and MiniRQLQ questionnaires. Allergy 2007; 62: 1372-1378.

24. Laforest L, Bousquet J, Pietri G, Sazonov KoceVAR V, YIN D, PACHECO Y et al. Quality of life during pollen season in patients with seasonal allergic rhinitis with or without asthma. Int Arch Allergy Immunol 2005; 136: 281-286.

25. Kiebert G, Sorensen SV, Revicki D, Fagan SC, Doyle $\mathrm{JJ}$, CoHEN $\mathrm{J}$ et al. Atopic dermatitis is associated with a decrement in health-related quality of life. Int J Dermatol 2002; 41: 151-158.
26. Osman LM, Calder C, Robertson R, Friend JA, LEgGe JS, Douglas JG. Symptoms, quality of life, and health service contact among young adults with mild asthma. Am J Respir Crit Care Med 2000; 161 (2 Pt 1): 498-503.

27. Laforest L, Bousquet J, Neukirch F, Aubier M, PieTRI G, Devouassoux G et al. Influence of sociodemographic factors on quality of life during pollen season in seasonal allergic rhinitis patients. Ann Allergy Asthma Immunol 2005; 95: 26-32.

28. Vilagut G, Valderas JM, Ferrer M, Garin O, LóPEz-GARCía E, ALONSO J. Interpretación de los cuestionarios de salud SF-36 y SF-12 en España: componentes físico y mental. Med Clin (Barc) 2008 24; 130: 726-735. SF12.

29. Katz PP, Yelin EH, Eisner MD, Earnest G, Blanc PD. Performance of valued life activities reflected asthma-specific quality of life more than general physical function. J Clin Epidemiol 2004; 57: 259-267.

30. Valero A, Ferrer M, Sastre J, Navarro AM, MonClús L, Martí-Guadaño E et al. Anew criterion by which to discriminate between patients with moderate allergicrhinitis and patients with severe allergic rhinitis based on the allergic rhinitis and its impact on asthma severity items. J Allergy Clin Immunol 2007; 120: 359-365.

31. Bousquet J, Van Cauwenberge P, Khaltaev N. Aria Workshop Group; World Health Organization. Allergic rhinitis and its impact on asthma. J Allergy Clin Immunol 2001 Nov; 108 (5 Suppl): S147-334.

32. Bousquet J, Neukirch F, Bousquet PJ, Gehano P, KLOSSEK JM, LE GAL M et al. Severity and impairment of allergic rhinitis in patients consulting in primary care. J Allergy Clin Immunol 2006; 117: 158-162. 
